Introduction People at the end of life (and their carers) require support on a 24/7 basis. Access to appropriate, immediate advice is important to optimse the quality of care, reduce anxieties and where appropriate avoid hospital admission. Use of video links to the home may allow such services to be delivered in a scalable and affordable way.

Aims To develop reliable means of deploying high quality video links between hospices and a person's own home or residential care setting.

Methods Using standards based, resilient videoconferencing, running over domestic broadband, links were established between a hospice and patients receiving support at home and in a range of residential care settings. The service also linked patients to the local acute hospital's teleconsultation centre. Staff at the hospice and patients/carers were able to both make and receive calls at any time. Proof of concept outcome measures included the ability to create a stable video link in a domestic setting and identification of clinical use cases where video enabled safe, appropriate care delivery. Qualitative indications of patient, carer and staff satisfaction with the approach were also recorded.

Results Where broadband speed exceeded $500 \mathrm{~kb} / \mathrm{s}$ (up and download) we established reliable video connexions in all cases. Service users were able to receive general symptom control and treatment advice, it was possible to avoid need for admission or to expedite admission when necessary. Staff and patient satisfaction with the approach was high, averaging 8.5/10 across a range of measures. The service is expanding to include links to all local Nursing Homes and to allow on-call palliative care consultants to join calls from their own home.

Conclusion Use of video-consultation to the home enables immediate delivery of palliative care support without need for travel and may avoid the need for some hospital admissions. The approach is valued by users.

\section{INSPIRING COMPASSIONATE CARE IN AN ACUTE HOSPITAL SETTING - THE POWER OF QELCA}

${ }^{1}$ Robert Standfield, ${ }^{1}$ Val Wellings, ${ }^{2}$ Alison Harrison, ${ }^{2}$ Jennifer Garside; ${ }^{2}$ St. Richard's Hospice, Worcester, United Kingdom, ${ }^{2}$ Worcestershire Acute Hospitals NHS Trust

\subsection{6/bmjspcare-2013-000591.10}

Transforming the traditional delivery of specialist end-of-life care education and training for nursing staff in acute hospital settings through the Quality End of Life Care for All (QELCA) education and training programme has resulted in immediate positive benefits for both the hospice and the local acute hospital Trust.

The prevalence of a top-down target driven culture has meant that persuading hospital managers in hospital settings that compassionate care is directly relevant to improving acute care has been a challenge. The opportunity to transform this thinking was taken by the local acute hospital when they embarked on the Transforming End of Life Care in Acute Hospitals Programme that includes the adoption of the AMBER care bundle and incorporates QELCA training.

Through the delivery of the prescribed QELCA curriculum, that combines and integrates theory with experiential learning through working alongside experienced hospice nurses, there has been a transformational change in the nursing care. This is underpinned by changed thinking and delivery of care that focuses directly on the needs of the patient rather than the needs of the organisation.
The impact in the Acute trust has included:

- $82 \%$ of relatives said they were aware that their relative's recovery was uncertain.

- $91 \%$ of patients received care supported by the AMBER care bundle had a patient and family meeting.

- $1.5 \%$ of patients who received care supported by the AMBER care bundle, who were discharged were admitted back to the hospital in an emergency.

- $100 \%$ patients had a DNACPR Form completed.

Successful partnership working has also resulted in a renewed interest in furthering specialist palliative and end-of-life care education within secondary care and the CCG extending the AMBER care bundle/QELCA as part of their CQUIN investment. Further funding for the QELCA training has been provided through a successful bid to the Strategic Health Authority.

\section{A HOSPICE EXPERIENCE OF PATIENT-LED ASSESSMENTS OF THE CARE AND ENVIRONMENT}

${ }^{1}$ Pauline Flanagan, ${ }^{1}$ Nicci Williamson, ${ }^{2}$ Ruth Grocott, ${ }^{2}$ Geoff Scaife; ${ }^{1}$ Douglas Macmillan Hospice, Stoke-on-Trent, UK, ${ }^{2}$ Carers Forum, Douglas Macmillan Hospice, Stoke-on-Trent UK

10.1136/bmjspcare-2013-000591.11

Background/context In April 2013, the Department of Health (DH) introduced patient-led assessment of the care environment (PLACE) to give patients a strong voice in the assessment of privacy, dignity, food and cleanliness in hospitals offering NHSfunded care. Carers are eligible to undertake this assessment on behalf of the patient.

The DH and NHS Commissioning Board recommend that hospices participate in this initiative, which provides a non-technical view of the buildings and non-clinical services and is based on a visual assessment against defined criteria and guidance. Aims

- To undertake the first PLACE assessment of the DMH.

- To use members of the Carers' Forum to undertake an assessment of the organisation.

Approach used The Carers' Forum members were invited to complete the PLACE assessment. Those involved needed to be objective, unbiased and have the confidence to be open and honest.

Two carers agreed to undertake the assessment, accompanied by the Clinical Governance Manager (CGM) and the deputy Infection Control Nurse; this gave the required PLACE Team ratio of patient to staff assessors.

The carers were classified as volunteers, underwent a standard $\mathrm{CRB}$ check and were issued with a name badge.

All assessors undertook training provided by the Health and Social Care Information Centre (HSCIC).

PLACE scoring was made against standard criteria and reflected what was seen on the day.

Outcomes DMH results were published on the HSCIC website.

The hospice is working with the PLACE team to make the assessment more applicable to hospices.

The carers felt that they were contributing to the quality process in a positive and constructive way.

Application to hospice practice Participation in a national initiative to measure quality from a patient/carer perspective. 